\title{
The Effect of Menu Type and Task Complexity on Information Retrieval Performance
}

\author{
Herre van Oostendorp ${ }^{*}, \mathrm{a}$, R. Ignacio Madrid ${ }^{\mathrm{b}}$ and Mari Carmen Puerta Melguizo ${ }^{\mathrm{a}}$ \\ ${ }^{a}$ Center for Content and Knowledge Engineering, Institute of Information and Computing Sciences, Utrecht University, \\ The Netherlands \\ ${ }^{b}$ Department of Experimental Psychology and Physiology of Behaviour, University of Granada, Spain
}

\begin{abstract}
Success in finding information in a website depends not only on the complexity of the information retrieval task per se but also on the way information is made accessible. We explored the role of navigation menus when performing information retrieval tasks. We compared expandable menus with sequential menus. Expandable menus preserve the full context of choice while the user is browsing. Sequential menus provide only partial context. Task complexity was manipulated along two dimensions: path length and path relevance. Path length leading to the target information was varied using different levels of depth. Path relevance - based on semantic similarity - is the relevance of the optimal navigation path to the search task. In general, results show benefits of presenting expandable menus giving fully contextual information when retrieval tasks are highly complex. More specifically, with expandable menus, highly complex retrieval tasks with high path length and low path relevance are more efficiently performed than with sequential menus. Furthermore, users are also less disoriented with expandable menus. A practical implication is that for complex websites path relevance and expandable navigation menus are important usability considerations.
\end{abstract}

\section{INTRODUCTION}

Gathering information is a strategic activity that nowadays can (at least in principle) be made much easier by retrieving information from Web pages. Users retrieve information using searching and/or browsing tools. When users browse they make navigational choices through the information space that has a structure defined by its linking connections (hyperlinks). This structure is however not shown explicitly to the users, so navigation in complex websites is frequently not an easy task and can lead to problems such as cognitive overload or disorientation $[1,2]$. Actually, disorientation and lostness are major problems affecting information retrieval on the WWW. Disorientation increases task performance time, and results in lower task success and higher error rate, e.g. more clicks to find the information $[3,4]$.

The effect of system variables such as the structure of the Web or more locally, of a hypertext, has been frequently examined (e.g. [2]). The use of hierarchical navigation menus or indexes and the depth and breadth of hierarchical menus have been recognized as the main variables affecting task performance $[5,6]$. Also, previous research has examined the effects of different menu types (i.e. expandable menus vs sequential menus within the category of hierarchical menus) on information retrieval performance [7, 8], finding contradictory results. These contradictory results when expandable and sequential menus are compared, may be related to task characteristics such as task complexity, a

*Address correspondence to this author at the Center for Content and Knowledge Engineering, Institute of Information and Computing Sciences, Utrecht University, the Netherlands; E-mail: herre@cs.uu.nl variable that has not being properly controlled in these studies. Task complexity is however an important variable that is nowadays getting more attention in the literature (e.g. $[9,10])$.

In this paper, we present the results of an experiment in which we compared the use of expandable versus sequential navigation menus on performing information retrieval tasks where task complexity was manipulated. In the next sections, a further elaboration of menu types and task complexity is presented. Then the experiment and the results we found are explained in detail. Finally we will discuss our results and draw conclusions.

\section{MENU TYPE: EXPANDABLE MENUS VS SEQUENTIAL MENUS}

A main factor affecting information retrieval success are the navigation tools or menu types used [11]. In this paper we compare two different menu types: expandable menus and sequential menus $[7,8]$ (see Fig. 1).

Navigation menus using expandable menus preserve the full context of the choice within the hierarchy, showing the full tree of past selections. Sequential menus, on the other hand, only display elements in the same level of the hierarchy, and the context is only partially shown in the form of 'breadcrumbs' that show the history of selections through the different Website levels (path breadcrumbs) or the location of the user within the site hierarchy (location breadcrumbs). Location breadcrumbs have been popularized in large Web directories like the search engine Yahoo. They mark the specific location of the user, often by using a list of links to represent the location of the current page. A number of studies have shown that they can lead to more efficient 

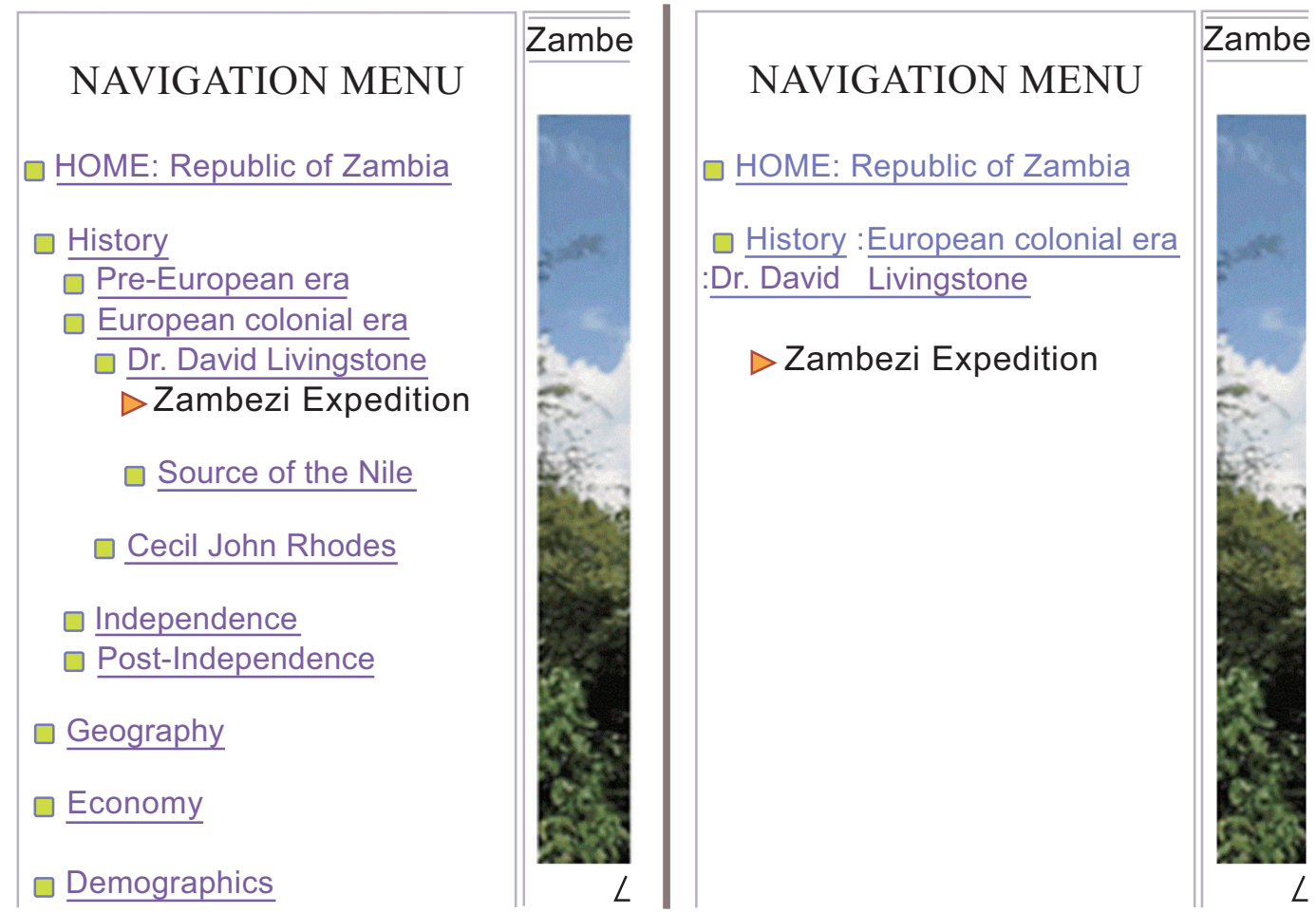

Fig. (1). Expandable menu (left) and sequential menu (right).

site navigation and improved user satisfaction compared to no-menu or static menus, particularly for large and hierarchical sites [12-14].

Kurniawan et al., [7] found that users working with the expandable menus showed a more optimal path to the target; that is expandable menus resulted in less deviations from the optimal clicks to arrive to a target. They did not find a significant difference in traversal time; that is, the total time needed to arrive at the correct page, between the two types of menus did not differ.

Zaphiris et al., [8] also compared the two types of navigation menu presentation. They also predicted that expandable indexes would result in a superior performance since they provide more context information. However, they found the opposite result; participants using sequential menus got faster response times, particularly at deeper levels of the hierarchy. The efficiency of information retrieval, measured as the number of clicks made above the optimal path, was similar for higher levels of the two menu types, but better for deeper levels of sequential menus. The authors suggested that the long lists of items in the expandable menu condition they manipulated could be responsible of this pattern of data, since with this detailed level of hierarchy users had to scan or even scroll for the context information. Task complexity can be another factor that could explain these inconsistent results.

\section{TASK COMPLEXITY}

The effect of information retrieval task characteristics on the navigation process has been studied and task complexity has been recognized as one of the important factors that affect the efficiency of information seeking strategies
[15-17]. Task complexity can be conceptualized from different approaches and measured using different methods. Campbell [18] described three general approaches: psychological (subjective), person-task interaction and objective (defined by task characteristics). Gwizka and Spence [9] explored the relationships between subjective and objective task complexity, and proposed to assess the complexity of information retrieval tasks in an objective manner, by breaking it down into three objective factors:

1. Path Length: the length of the navigation path leading to the target information. It indicates the depth or level of the target information in the hierarchical information structure in the web site. We will use in this study a maximal depth of four levels.

2. Page Complexity: the complexity of navigation choices on each web page (i.e., visual design, number of link labels, etc).

3. Page Information Assessment: the difficulty of relevance judgment on pages that contain the desired information (See [9]).

We add to these components of task complexity the concept of Path Relevance: the degree to which the description of the task is pointed out by the optimal path to the target information. For some tasks it is very clear what the optimal path is through the overlapping in meaning between the task's goal and the index names used in the website, for other tasks this is less clear. A path highly relevant to the task description contains a high overlap in meaning (see an example in section Method), and this is what we called high Path Relevance. 
In our study we examined both Path Length and Path Relevance to study task complexity, while we kept the Page Complexity of the web pages and Page Information Assessment constant. Both variables represent different levels of task complexity. Path Length is related with more structural aspects of the Web site and can be easily measured. Path Relevance refers to semantic aspects of the information during search navigation and requires measurement of the semantic similarity between the optimal search path and the goal as described in the task.

The reason to include Path Relevance in the current study is based on the cognitive model CoLiDeS of web navigation $[19,20]$. This model simulates a website visitor's web navigation process, and it involves a comprehension model based approach that uses Latent Semantic Analysis to compute the degree of semantic similarity ("information scent") between user goals statements and screen objects (mainly link labels). CoLiDeS explains how users parse and comprehend the content of a web page and then select what action (click) to perform next. This comprehension process is influenced by the user's goal (task) and background knowledge. CoLiDeS compares the user's goal with link texts (labels) on web pages and selects the link that best matches the user's goal. The selected link is clicked on and the process of judging link relevance (information scent), and selecting a link is repeated until the user's goal is attained or the user stops. The relatedness of screen objects to the user's goal is measured, mainly, on basis of semantic similarity.

Van Oostendorp and Juvina [21] developed their model, CoLiDeS+, based on extensions of the CoLiDeS model. Particularly, the concept of "path adequacy" was introduced as a complement to the concept of information scent and appeared to play a significant role in predicting web navigation of users. Path adequacy is the semantic similarity between the navigation path (the succession of links that have been selected prior to a particular moment) and the user's information retrieval goal. Path adequacy reflects the goal-relevance of past selections and integrates in this way the preceding context to current link choice. Semantic similarity can be measured using Latent Semantic Analysis [22]. We propose that path adequacy can also be used to measure the complexity of a certain information retrieval task in terms of Path Relevance, by computing the semantic similarity between user goal and the optimal navigation path leading to the target information. However, path adequacy characterizes what users do, while Path Relevance characterizes a task, i.e. its overlap in meaning with the optimal path. The rationale of this proposal is that if a task does not properly fit to the link labels of the navigation menu that have to be followed to find the target information (low Path Relevance), the information retrieval task is more difficult and, consequently, should have negative effects on navigation behaviour and performance.

\section{RESEARCH OBJECTIVES}

Results of the studies of Kurniawan et al., [7] suggested that menu type can have an effect on information retrieval performance. A menu type such as expandable menus can provide information about where a user is in relation to the information structure as a whole, and thereby facilitate navigation and finding information. We will use in our study the sequential menu as reference (control) condition because - as indicated above- the empirical research literature is clear about the value of this kind of index, and examine whether expandable menus are even better. The benefit of expandable menus giving more contextual information compared to sequential menus can even be bigger as task complexity increases, at least when the menu is clearly visible on screen within the screen border. Task complexity is here measured on basis of Path Length and Path Relevance. We propose that both factors have an influence on task complexity: low complex tasks are characterised by short Path Length and High Path Relevance, and highly complex tasks by long Path Length and low Path Relevance. The usefulness of expandable menus could be higher with high complex tasks, since users can take advantage of the contextual information to guide their navigation. Consequently, our main hypothesis is that participants using expandable menus achieve a better task performance than participants using sequential menus particularly when performing complex tasks, while the difference is much smaller when performing simple tasks. We will examine participant's performance by measuring the accuracy of their performance on the information retrieval tasks, as well as by measuring the time needed to find an answer. Furthermore, we measured the objective lostness and perceived disorientation of participants. The first approach implies to infer participant's level of lostness from analyzing participant's actions when browsing [23, 24]. Using this approach one can for instance calculate the number of deviations form the optimal pathway. Other authors however have claimed that instead of inferring lostness from participant's actions, it is better to directly ask user to indicate feelings regarding disorientation, using a questionnaire [5]. We will use both kinds of measures and hypothesize that lostness and perceived disorientation will be higher for participants using the sequential menus than for participants using the expandable menus, also particularly for more difficult tasks. We expect that participants using expandable menus will actually be less disoriented, and also feel themselves less disoriented than participants using sequential menus, again particularly when performing complex tasks.

\section{METHOD}

\section{Participants}

Thirty-five bachelor or master students (24 males and 11 females) at the University of Utrecht took part in this study. The mean age of the participants was 20.54. They received a 7.5 Euro book-gift card. We can assume that for these participants prior knowledge of the hypertext materials (information about Zambia) will be low.

\section{MATERIALS}

\section{Website}

The topic of the Web site used in the experiment was "Discovering Zambia" (8936 words long). Webpage contents were adapted from general Wikipedia articles about the history, geography and culture of Zambia, and were divided into 36 pages. The length of the pages was controlled to be about the same with a mean length per page 
of 255 words ( $\mathrm{sd}=53$ ). All the pages included text and one picture, and they could be read without scrolling.

In the process of constructing the Website structure, two of the authors of this paper rated the similarity between the different pages, and their ratings were used for organizing the website hierarchy. For every page they selected the 8 most related pages and made a ranking from 1 to 8 . The average ratings were used to cluster the most related content, which defined the web site hierarchy and the structure of the navigation menu. The maximum depth of the hierarchy was 4.

There were two different тепи types used in the study: an expandable menu and a sequential menu with location breadcrumbs (see Fig. 1). The WWW browser used in this study did hide the tools bar, so participants could only navigate using the webpage hyperlinks, not the back and forward buttons or other browser features.

\section{Information Retrieval Tasks: Path Relevance and Path Length}

We were interested in manipulating task complexity along two dimensions: Path Relevance and Path Length. First, we constructed a pool of 45 questions covering all page contents at different depths in the hierarchy. The question statements were considered to reflect the user goal. Second we use the Encyclopedia corpus available at the LSA website [25] to compute the LSA values between the description of all questions and their corresponding optimal path link labels, that is, the Path Relevance of the optimal path, and they were assigned to two sets of Path Relevance values (low and high) using the percentile 25 and 75 as the cut-off. Examples of tasks of low and high Path Relevance are:

For a LOW Path Relevant task:

Zambia holds unique wild life in their natural reserves. Which is a unique mammal that you can find at the Luangwa Valley?

Optimal path with the following links: Zambia > Economy $>$ Tourism and Safari $>$ South Luangwa Park $>$ Giraffe $($ LSA $=0.00)($ Level 4$)$

For a HIGH Path Relevant task:

In August 1865, David Livingstone sailed for Africa to seek the sources of the Nile. Which river did he confuse with the "real" Nile"?

Optimal path with the following links: Zambia > History $>$ European Colonial Era $>$ David Livingstone $>$ Source of the Nile $($ LSA $=0.49)($ Level 4)

Finally, we extracted 12 tasks from these two sets (6 high, 6 low relevant tasks) that covered the 2, 3 and 4 levels of Path Length (menu depth) to be used in our experiment.

\section{Measures}

As dependent variables we used several measures that were extracted from users' session logs and their responses to the questionnaires filled out during the experiment. For each of the 12 information retrieval tasks we recorded:

Task performance: It was measured with two variables:
Task accuracy: It was the score on the information retrieval tasks. Participants who found the correct answer to the task before the time limit scored 1 . When the participant's answer was incorrect but it was extracted from the page that contained the correct answer it was scored 0.5. Incorrect answers from incorrect pages, answers above the time limit or no answers were scored 0 .

- $\quad$ Task response times: It was the time needed to find the answer in seconds. The time started when the home page was shown and ended when the participant wrote the answer in the response box.

Disorientation: Two measures of disorientation were used.

Objective measure: Lostness. It was measured by using a [23]' $\mathrm{L}$ measure, that is conceptualized in terms of number of deviations from the optimal pathway.

$\mathrm{L}=\sqrt{ }\left((\mathrm{N} / \mathrm{S}-1)^{2}+(\mathrm{R} / \mathrm{N}-1)^{2}\right)$

Where:

$\mathrm{R}=$ number of nodes required to finish the task successfully (thus, the number of nodes on the optimal path);

$\mathrm{S}=$ total number of nodes visited while searching;

$\mathrm{N}=$ number of different nodes visited while searching.

With L, we obtain an objective measure of disorientation based on participants' behaviour. However [3], claimed that disorientation can be better characterized by asking users directly. For this reason we also tested perceived disorientation at the end of the experiment.

Subjective measure: Perceived disorientation. Participants filled out the self-perceived disorientation questionnaire, based on 5-point Likert scales, validated by [3] at the end of the experiment. Perceived disorientation has been identified as a key construct in measuring the quality of humancomputer interaction, and extra support to the psychometric properties (reliability and validity) of this scale have been provided by [26, 27]. This questionnaire included statements such as "Navigating between pages was a problem" or "I didn't know how to get my desired location" $(1=$ strongly disagree to $5=$ strongly agree $)$. The mean score on the 7 questions of this questionnaire was used as an overall index of disorientation during the whole session.

\section{Procedure}

First, participants received the general instructions of the experiment and were presented with a computerized questionnaire to enter their personal data (gender, age, email, etc.). After that, the information retrieval tasks began. One task per time was shown, for which the participants had to search for the answer in the website. During the information retrieval task the question was shown on the top of the screen followed by an empty box to type the answer when it 
was found. Participants were told to do it as quickly and accurately as they could, since there was a time limit of four minutes to find the answer. After all the retrieval tasks were completed they filled out the perceived disorientation questionnaire.

\section{RESULTS}

\section{Task Accuracy}

The percentage of participants that were unable to complete accurately each of the tasks ranged between $71 \%$ on a task characterized by High Path Length and Low Path Relevance and $0 \%$ on a task characterized by High Path Length and High Path Relevance.

A mixed $2 \times 2 \times 2$ ANOVA analysis was used with Path Length, Path Relevance and Menu Type as independent variables and the mean accuracy score on the information retrieval tasks as dependent variable. Results showed a main effect of Path Length $(\mathrm{F}(2,66)=41.28, \mathrm{p}<.001)$, the accuracy decreased as Path Length increased. A main effect of Path Relevance was also significant $(\mathrm{F}(1,33)=12.46, \mathrm{p}<.01)$, the accuracy was higher in the high Path Relevance tasks. The effect of Menu Type did not reach statistical significance $(\mathrm{p}>.05)$. Finally, the interaction between Path Length and Path Relevance was significant $(\mathrm{F}(2,66)=6.04, \mathrm{p}<.01)$. Other effects were not significant ( $p>.05)$. In Fig. (2) the relationship between Path Length and Path Relevance is shown. Simple analyses indicated that the differences between levels of Path Relevance were only significant in tasks with Path Length 3 or 4, but not in Path Length 2 tasks. This means that particularly for longer paths low relevant paths lead to lower accuracy.

\section{Task Response Times}

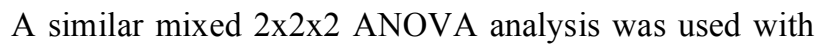
Path Length, Path Relevance and Menu Type as independent variables and task response times as dependent variable. For this analysis we only considered the response times to tasks on which the users gave the correct answer.

The main effect of Path Length was strongly significant $(\mathrm{F}(2,66)=108.67, \mathrm{p}<.001)$, response times increased as Path Length increased. There was also a strong and significant main effect of Path Relevance $(\mathrm{F}(1,33)=45.59, \mathrm{p}<.001)$, response times to low Path Relevant tasks were higher than for high relevant paths. The main effect of Menu Type was not significant but there was a significant interaction effect between Menu Type x Path Length $(\mathrm{F}(2,66)=12.67, \mathrm{p}<.001)$ and Path Length $x$ Path Relevance $(F(2,66)=7.99, p<.001)$ was also significant, furthermore Menu Type $x$ Path Length $x$ Path Relevance $(F(2,66)=6.75, p<.01)$ was also significant. In Fig. (3) we depicted the means. For high Path Relevant tasks there were no differences between menu types, only response times increased there as Path Length did. But for low Path Relevant tasks the expandable menu did lead to faster responses than the sequential menu when the path length was high (Path Length 4), for shorter paths there were no significant differences between the two menu types.

\section{Objective Measure: Lostness}

A similar mixed ANOVA analysis was used with Path Length, Page Relevance and Menu Type as independent variables and the objective lostness measure as dependent

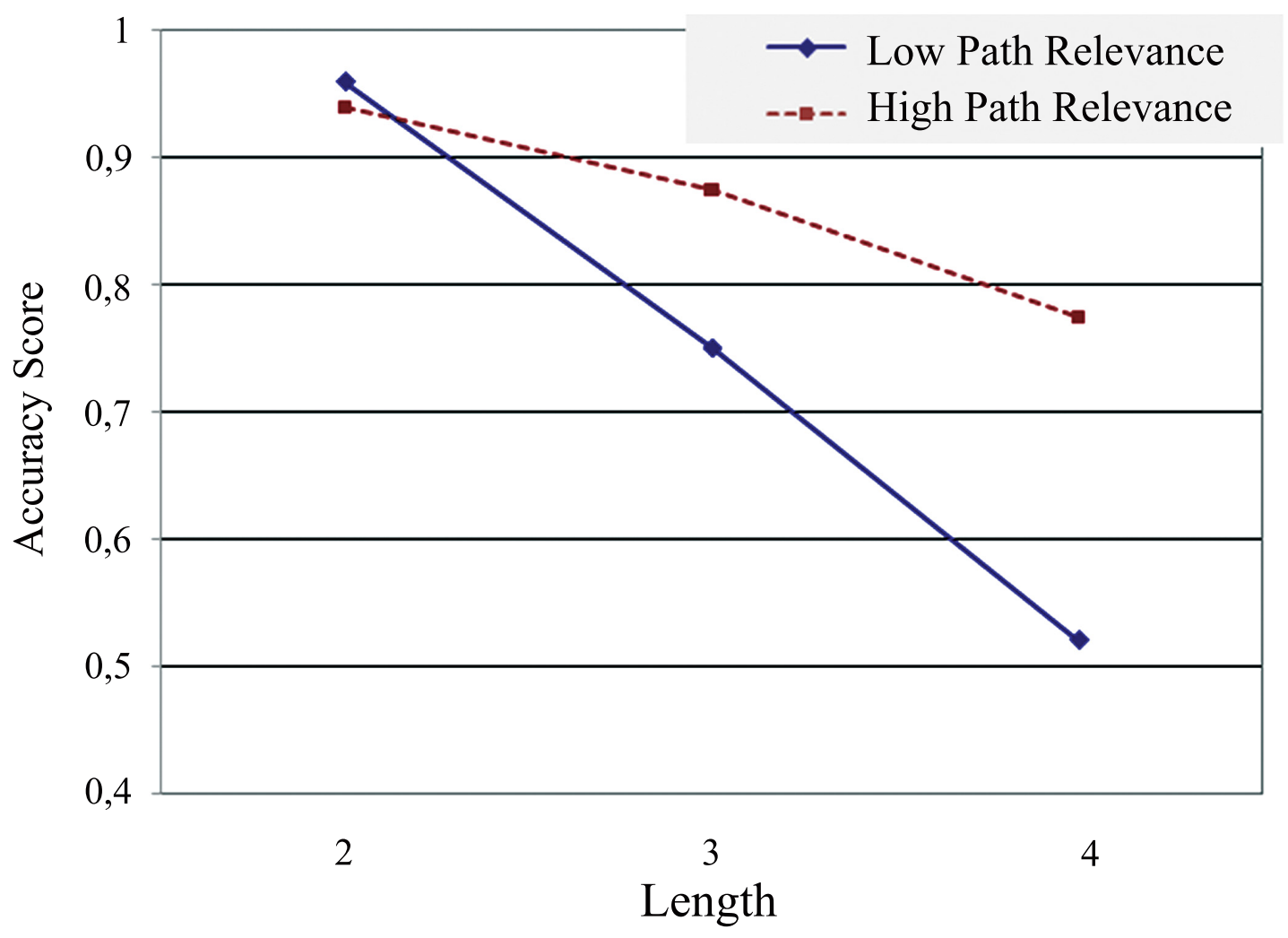

Fig. (2). Mean accuracy in relation to path length and path relevance. 


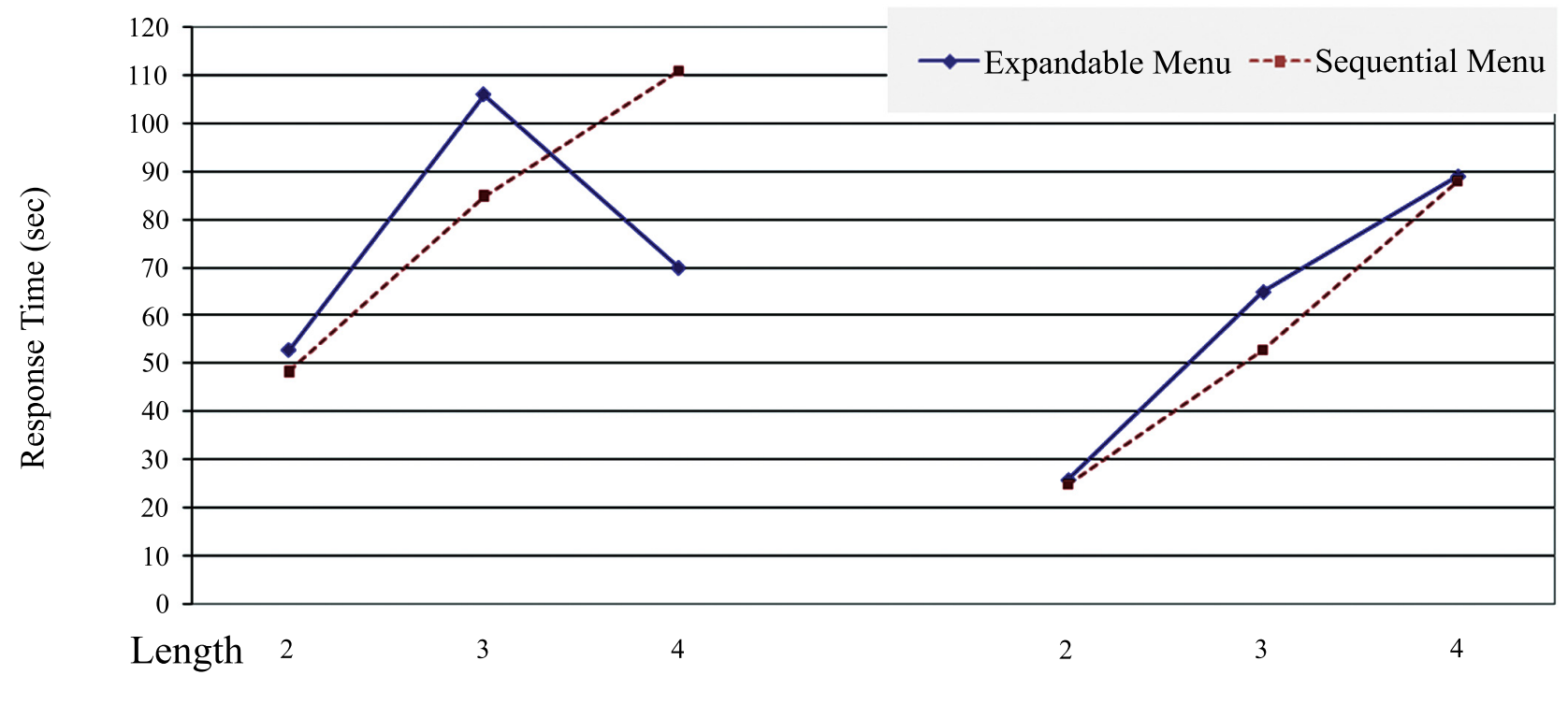

Low Path Relevance

High Path Relevance

Fig. (3). Mean response time in relation to path length, path relevance and menu type.

variable. There was a significant main effect of Menu Type $(\mathrm{F}(1,33)=8.88, \mathrm{p}<.01)$, a main effect of Path Length $(\mathrm{F}(2,66)=37.73, \mathrm{p}<.001)$ and a strong main effect of Path Relevance $(\mathrm{F}(1,33)=317.42, \mathrm{p}<.001)$. There was also an interaction effect between Path Length and Menu Type $(\mathrm{F}(2,66)=5.17, \mathrm{p}<.01)$ and between Path Length and Path Relevance $(\mathrm{F}(2,66)=14.60, \mathrm{p}<.001)$. Finally, there was a significant third order interaction Menu Type $x$ Path Length $x$ Path Relevance $(F(2,66)=3.11, p<.05)$. The sequential menu induced more lostness, particularly in tasks characterized by longer Path Length and a low Path Relevance (Fig. 4).

\section{Subjective Measure: Perceived Disorientation}

A t-test was performed using Menu Type as independent variable on the mean overall scores on the perceived disorientation questionnaire. There was a significant effect of Menu Type $(\mathrm{t}(33)=2.05, \mathrm{p}<.05)$. Participants using the sequential menu felt more disoriented than those using the expandable menu (sequential menu: mean $=3.70, \mathrm{sd}=.86$ and expandable menu: mean $=3.09$, $\mathrm{sd}=.89$ ).

\section{CONCLUSION AND DISCUSSION}

This research has focused on the effects of task complexity and different menu types on task performance (accuracy and response time) and disorientation of users during navigation. Summarizing the main results: task complexity measured by Path Length and Path Relevance strongly influenced the results on navigation performance and retrieval task success: when Path Length decreases and Path Relevance increases, task accuracy improves and response time is shortened. Also significant effects were found on lostness. Concerning the effects of Menu Type we did find significant interaction effects on response times: particularly for deeper levels and lower Path Relevance expandable menus had a positive effect. There was not a significant effect of Menu Type or interaction effect on accuracy. Correspondingly particularly for deeper levels and lower Path Relevance we did find significantly less lostness with expandable menus. Also on perceived disorientation menu type had a significant effect.

As hypothesized, we found that information search task complexity, as measured by Path Length and Path Relevance, negatively affected task performance. Results showed that in general task accuracy increased and response time decreased as Path Length decreased and Path Relevance increased. On lostness we found similar and strong effects: a longer path and lower relevance resulted in more lostness during navigation. Returning to the aspects of task difficulty mentioned in the Introduction section that were distinguished by [9] and [10], our results support the importance of Path Length as factor relevant to task difficulty. These results also confirm the idea that not only structural but also semantic aspects such as Path Relevance are important in web navigation [21].

Concerning the effect of Menu Type we expected in line with [7], that expandable menus would result in a more optimal path to the target information and better performance because of the increased amount of contextual information available during search compared to sequential menu that provides only limited context. Although we did not found effects of menu type on performance, our results show that for low Path Relevance tasks the expandable menu did lead to faster responses than the sequential menu when the path length was high (Path Length 4). Perhaps the difficulty of the current retrieval tasks was not high enough to find direct effects of Menu Type on accuracy. We are currently performing a follow-up study in which we introduced an even more difficult task; tasks where participants had to find 


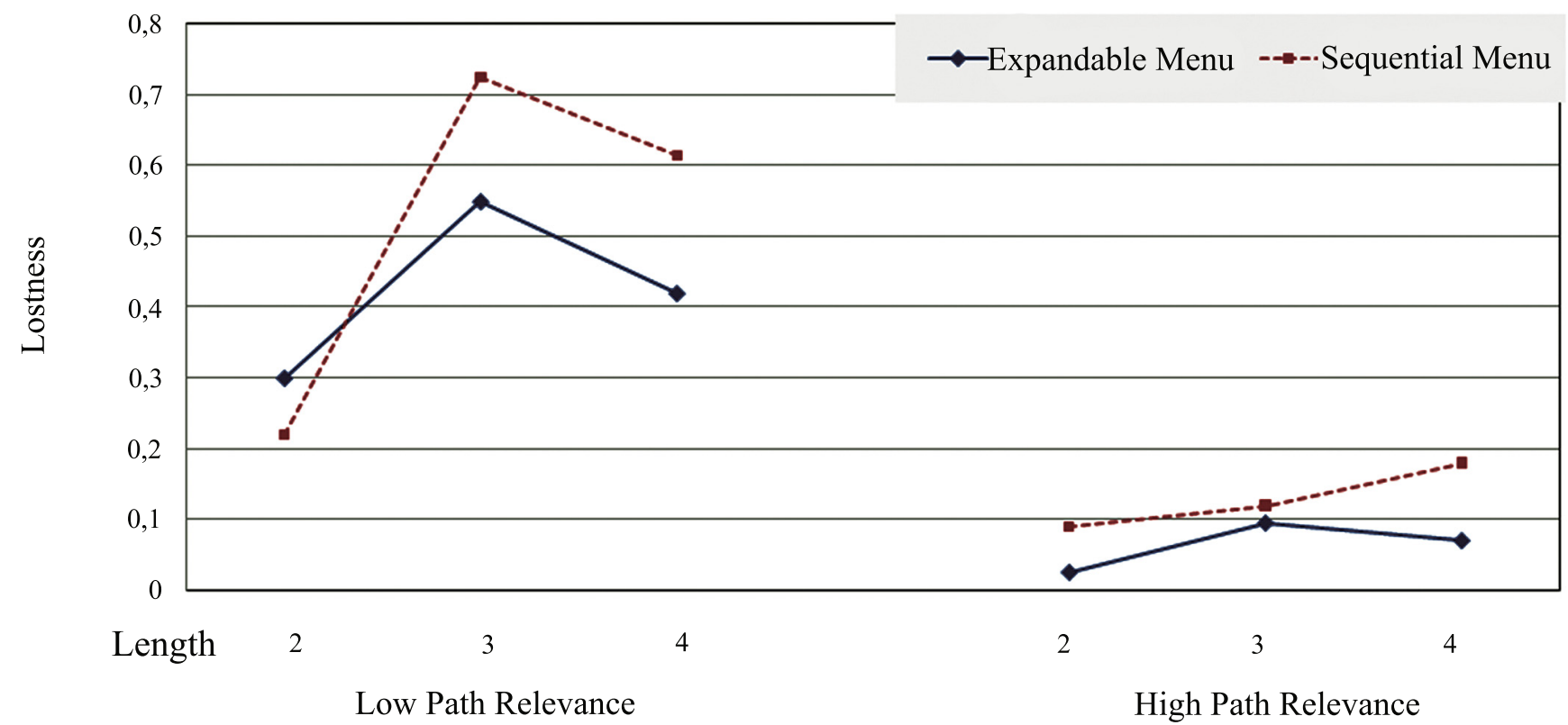

Fig. (4). Mean lostness in relation to path length, path relevance and menu type.

and combine information located on different places on a page. We expect to find under these circumstances a main effect of Menu Type on performance in terms of accuracy.

In relation to lostness, expandable menus as hypothesized were found to produce less perceived disorientation than sequential menus when assessing the search and navigation session as a whole afterwards. However, results from the objective lostness measures showed that this effect was mediated by task complexity: lostness was high for participants using sequential menus, particularly when Path Length was longer and Path Relevance low.

In general, the results we found support our main hypothesis that expandable menus, at least when they are fully and clearly visible on screen, are more beneficial for information retrieval tasks particularly when the complexity is high. It seems that in high complex tasks users can take advantage of the context information provided by the expandable menus to guide their navigation, and subsequently they reduce their lostness, need less time to perform the tasks and feel less disoriented. The results even indicate a threshold effect of task complexity (see Figs. 2-4): only when the task is really difficult (Path Length at least two steps and Path Relevance low) we do find positive effects of expandable menus on retrieval performance

A practical implication can be extracted from this study. Expandable menus can enhance the usability of a web site, and are thus useful to include. However, in website design, especially for large and complex websites, it is not always possible to keep the number of levels of the hierarchy low, resulting in a menu within the screen border which seemed to be the problem in the study by [8]. Especially in those cases, more attention has to be directed to enhance the Path
Relevance; that is, optimizing the information scent of the ideal menu path to the search goal. Frequently occurring search tasks can be checked beforehand on Path Relevance. When it appears that for these tasks the relevance is low, the organization of information or formulation of the link labels should be changed. The advantage is that these checks can be done before real users are exposed to the web site. This topic falls into the field of usability studies that use cognitive models for predicting navigation problems and enhancing web navigation (see further [19-21, 28]. Based on the above mentioned CoLiDeS model, these authors were able to identify and repair web usability problems when they applied the 'Cognitive Walkthrough for the Web' usability inspection method. Van Oostendorp and Juvina [21] did show that model-generated navigation support, by highlighting links that were relevant to the user's goal, had positive effects on satisfaction, navigation and task performance. The advantage of this model-approach is that it is psychologically valid because it is based on well-verified cognitive architecture and model's predictions are confirmed to be consistent with what real human users would do.

\section{REFERENCES}

[1] Conklin J. Hypertext: An Introduction and Survey. IEEE Comput 1987; 20 (9): 17-41.

[2] McDonald S, Stevenson RI. Disorientation in hypertext: The effects of three text structures on navigation performance. Appl Ergon 1996; 27 (1): 61-8.

[3] Ahuja JS, Webster J. Perceived disorientation: an examination of a new measure to assess web design effectiveness. Interact Comput 2001; 14: 15-29.

[4] Otter M, Johnson H. Lost in hyperspace: metrics and mental models. Interact Comput 2000; 13: 1-40.

[5] Jacko J A, Salvendy G. Hierarchical menu design: breadth, depth, and task complexity. Percept Mot Skills 1996; 8: 1187-201. 
[6] Larson K, Czerwinski M. Web page design: Implications of memory, structure and scent for information retrieval. Proceedings of CHI 98; 1998; New York: ACM Press. pp. 25-32.

[7] Kurniawan SH, Zaphiris P, Ellis RD. Comparing older and younger adults traversal time in expandable and non-expandable hierarchical structures. In: Proceedings of human factors and ergonomics society 46th Annual Meeting Baltimore, USA 2002; pp. 185-8.

[8] Zaphiris P, Shneiderman B, Norman KL. Expandable indexes vs Sequential menus for searching hierarchies on the World Wide Web. Behav Inf Technol 2002; 21 (3): 201-7.

[9] Gwizdka J, Spence I. What can searching behavior tell us about the difficulty of information tasks? A study of web navigation. Proceedings of the 69th Annual Meeting of the American Society for Information Science and Technology (ASIS\&T). vol 43. Medford, NJ: Information Today, Inc; 2006.

[10] Gwizdka J, Spence I. Implicit measures of lostness and success in web navigation. Interact Comput 2007; 19(3): 357-69.

[11] Chen C, Rada R. Interacting with hypertext: A meta-analysis of experimental studies. Human - Comput Interact 1996; 11: 125-56.

[12] Instone K. Location, path and attribute breadcrumbs. In: Proceedings of the $3^{\text {rd }}$ Annual Information Architecture Summit. Available from: http://keith.instone.org/breadcrumbs/ [Accessed on March 17, 2002].

[13] Teng H. Location breadcrumbs for navigation: An exploratory study. Unpublished Master's thesis, Faculty of Computer Science, Dalhousie University, NS, Canada, 2003.

[14] Blustein J, Ahmed I, Instone K. An evaluation of menu breadcrumbs for the WWW: Proceedings at the Sixteenth ACM Conference on Hypertext and Hypermedia (HT' 05). New York: ACM Press; 2005; pp. 202-204.

[15] Byström K. Information and information sources in tasks of varying complexity. J Am Soc Inf Sci Technol 2002; 53: 581-91.

[16] Hochheiser H, Shneiderman B. Performance benefits of simultaneous over sequential menus as task complexity increases. Int J Hum Comput Interact 2000; 12: 173-92.
[17] Vakkari P. Task complexity, problem structure and information actions. Integrating studies on information seeking and retrieval. Inform Process Manage 1999; 35: 819-837.

[18] Campbell, DJ. Task complexity: A review and analysis. Acad Manage Rev 1988; 13: 40-52.

[19] Kitajima M, Blackmon MH, Polson PG. A comprehension-based model of web navigation and its application to web usability analysis. In McDonald S, Waern Y, Cockton G. Eds. People and Computers XIV, Usability or Else! Proceedings of HCI 2000; Sunderland: Springer-Verlag. pp. 357-73.

[20] Kitajima M, Polson PG, Blackmon MH. CoLiDeS and SNIF-ACT: Complementary models for searching and sensemaking on the Web. Paper presented at the Human Computer Interaction Consortium (HCIC) Winter Workshop; 2007.

[21] Van Oostendorp H, Juvina I. Using a cognitive model to generate web navigation support. Int J Hum Comput Studies 2007; 65: 88797.

[22] Landauer TK, Foltz PW, Laham D. An introduction to Latent Semantic Analysis. Discourse Process 1998; 25: 259-84.

[23] Smith P. Towards a practical measure of hypertext usability. Interact Comput 1996; 8 (4): 365-81.

[24] Puerta Melguizo MC, van Oostendorp H, Juvina I. Predicting and Solving Web Navigation Problems. In: Hypertext 2007: Eighteenth ACM Conference on hypertext and hypermedia; 2007; pp. 47-48.

[25] Dennis S. How to use the LSA Web site. In: Landauer TK, McNamara D, Dennis S, Kintsch W, Eds. The Handbook of Latent Semantic Analysis. Mahwah, NJ: Erlbaum 2007; pp. 57-70.

[26] Van Schaik P, Ling J. Using online surveys to measure three key constructs of the quality of human-computer interaction in web sites: Psychometric properties and implications. Int J Hum Comput Stud 2003; 59: 545-67.

[27] Van Schaik P, Ling J. Five psychometric scales for online measurement of the quality of human-computer interaction in Web Sites. Int J Hum Comput Stud 2005; 18(3): 309-22.

[28] Blackmon MH, Kitajima M, Polson PG. Repairing usability problems identified by the cognitive walkthrough for the web: Proceedings of the Conference on Human Factors in Computing Systems; New York: ACM Press; 2001; pp. 497-504. 